\title{
MINIREVIEW
}

\section{Auxins as One of the Factors of Plant Growth Improvement by Plant Growth Promoting Rhizobacteria}

\author{
AMBREEN AHMED ${ }^{1 *}$ and SHAHIDA HASNAIN ${ }^{2}$ \\ ${ }^{1}$ Department of Botany, University of the Punjab, Lahore 54590, Pakistan \\ ${ }^{2}$ Department of Microbiology and Molecular Genetics, University of the Punjab, Lahore 54590, Pakistan
}

Submitted 29 July 2013, revised 12 November 2013, accepted 12 May 2014

\begin{abstract}
Plant growth promoting rhizobacteria (PGPR) promote plant growth by various mechanisms such as phytohormone production, enhanced water and nutrient uptake, improved nitrogen availability in the soil, production of ACC-deaminase for ethylene breakdown, phosphate solubilization, siderophore production etc. Microbial auxin production is the major factor not only responsible for strengthening the plant-microbe relationship but it also promotes plant growth and development in a positive manner. Thus, bacterial auxin production potential can be exploited for plant growth improvement that may be effective in reducing the hazardous effects of chemical fertilizers on the ecosystem used to obtain higher yields. The present review gives a better understanding of various factors and mechanisms involved in auxin production by PGPR that may be helpful in proper exploitation of these natural resources in a beneficial way.
\end{abstract}

Key words: auxin, IAA, PGPR, rhizobacteria, tryptophan

\section{Introduction}

Phytohormones are chemical signals produced by plants which coordinately control the growth and development of the plants at extremely low concentrations (Muller and Munne-Bosch, 2011; Muday et al., 2012). Plants follow particular developmental patterns determined and regulated by phytohormones. The sites of action and production of these phytoregulators are generally quite distant from one another (Avanci etal., 2010; Liu etal., 2012). According to the classical view, five major classes of phytohormones are auxins, cytokinins, gibberellins, ethylene and abscisic acid (Jamil et al., 2012; Robles et al., 2012) but there is increasing evidence of other compounds to have shown growth regulating activities in plants. Among these are brassinosteroids, jasmonates, salicylic acid, strigolactones, etc. (Taiz and Zeiger, 2010; Costigan et al., 2011; Koltai, 2011; Muller and Munne-Bosch, 2011; Facella et al., 2012). Auxins, cytokinins, gibberellins and brassinosteroids promote shoot growth while others including ethylene, abscisic acid and jasmonates control growth activities by regulating growth inhibitory processes in plants such as dormancy, abscission, senescence etc. (Acosta and Farmer, 2010; Dempsey et al., 2011; Costigan et al., 2011; Liu et al., 2012; Robles et al., 2012).

\section{Auxin: Master control phytohormone}

Among the various phytohormones, auxins act as a master control, regulating most of the plant processes, directly or indirectly, thus can be considered responsible for most of the developmental patterns in plants (Tanimoto, 2005; Wu etal., 2011). Generally auxins are produced in the meristematic areas of the plant stem although other plant parts, for instance, shoots, roots and leaves may also produce auxins. Auxin concentration varies depending on the influx and efflux from the tissues, its biosynthesis from tryptophan and formation of IAA conjugates (Tanimoto, 2005). Auxin levels are also affected by other phytohormones such as cytokinins, ethylene, gibberellins, jasmonates and brassinosteroids. The signaling crosstalks help in

\section{Abbreviation:}

PGPR - plant growth promoting rhizobacteria; IAA - indole-3-acetic acid; Trp - tryptophan; IPyA - indole pyruvic acid; IAM - indole acetamide.

\footnotetext{
* Corresponding author: A. Ahmed, Department of Botany, University of the Punjab, Lahore 54590, Pakistan; e-mail: ambreenahmed1@ hotmail.com
} 
coordinate regulation of various plant processes (Acosta and Farmer, 2010; Stewart and Nemhauser, 2010; Blomster et al., 2011; Koltai, 2011; Muller and Munne-Bosch, 2011; Chiu et al., 2012; Facella et al., 2012; Muday et al., 2012; Robles et al., 2012). Auxins and brassinosteroids have been found to interact with each other to regulate root development (Teale et al., 2008). Similarly, auxins regulate GA responses by affecting the stability of DELLA proteins (Teale et al., 2008; Pierik et al., 2009). Lower auxin levels caused by impaired auxin transport, leads to reduced GA synthesis due to the stabilization of DELLA proteins. GA plays its role in root gravitropism but is not essentially required for gravitropic responses (Willige et al., 2011). Auxins stimulate root formation and lateral root initiation. Cytokinins, on the other hand, suppress the formation of lateral roots by controlling cell differentiation in the root meristem (Teale et al., 2008). Therefore, plant development as a whole is dependent on the signaling crosstalks and interactions among various phytohormones and DELLA proteins in the plant environment determining the overall shape and growth of the plants (Grant and Jones, 2009).

Auxins regulate numerous plant processes. These play central regulatory role in cell elongation (Giehl et al., 2012; Chapman et al., 2012). Cell enlargement is the factor responsible for tropic responses mediated by auxins (Tanimoto, 2005). Auxin levels influence the perception of light by photoreceptors i.e., cytochromes which in turn affects the overall metabolism and biosynthetic pathways of plants (Facella et al., 2012; Kami et al., 2012). Other plant processes controlled by auxins include lateral root initiation, ethylene production, floral meristem initiation, vascular differentiation, apical dominance, embryo development, leaf abscission, parthenocarpy, differentiation of phloem and xylem, floral bud formation and fruit development (Hopkins and Huner, 2004; Smith, 2008; Facella et al., 2012; Muday et al., 2012; Niklas and Kutschera, 2012). Auxins stimulate the initiation of new leaves at the apical meristem. Cells present in the vicinity of growing leaves are generally depleted of auxins to restrict the formation of new leaves too close to each other. Greater amounts of auxins help the young leaves and fruits to remain intact whereas reduction in auxin levels in falling leaves and fruits results in the formation of abscission layer at the base of petiole or fruit stalk which is followed by fruit or leaf drop. Appropriate amounts of auxins are generally required for the proper development of floral parts i.e., style and stigma (Stewart and Nemhauser, 2010). Development of various plant organs from the embryo is also regulated by the gradient of auxins (Stewart and Nemhauser, 2010; Taiz and Zeiger, 2010). Vein patterning in plants is found to be affected by polar auxin transport (Smith, 2008; Donner et al., 2010). The effects of auxins, cytokinins and brassinosteroids on root growth are concentration dependent (Tanimoto, 2005; Muday et al., 2012). Auxins promote root growth up to an optimal level. Higher concentrations cause retardation of root development (Taiz and Zeiger, 2010; Facella et al., 2012). The growth inhibitory effects of higher concentration of auxins on plant roots are the consequence of increased ethylene synthesis stimulated by auxin levels via enhanced biosynthesis of ACC (aminocyclopropane carboxylate), immediate precursor of ethylene, as root growth inhibition by auxins is found to be reversed by the application of inhibitors of ethylene biosynthesis (Ruzicka et al., 2007; Muday et al., 2012). Ethylene is required during seed germination but high concentration of ethylene, after germination, may lead to inhibition of root growth. PGPR were found to inhibit the activity of ACC i.e., aminocyclopropane carboxylate (ACC) which acts as ethylene precursor thereby reducing ethylene synthesis which is responsible for inhibition of root growth (Glick, 1995; Glick et al., 1997; Glick et al., 1998; Glick 2005).

Plant responses observed with auxins include cell enlargement, cell division, ethylene production, increased protein and RNA synthesis and RNA polymerase activity (Taiz and Zeiger, 2010; Muday et al., 2012; Niklas and Kutschera, 2012). Auxins affect the metabolic activities of the cell by controlling the influx and efflux of ions through the plasma membrane or cell wall loosening, for which they bind to the transmembrane protein receptors such as ABP1 (Auxin-binding protein 1 ) at the cell surface. Auxins may also stimulate new patterns of gene expression by acting as a signal molecule triggering signal transduction pathways (Willige et al., 2011; Wu et al., 2011). These pathways may promote biosynthesis of transcription factors that result in the production of enzymes involved in catalyzing various metabolic activities. Auxin transporter molecule (AuxI) in the plasma membrane facilitates the active transport of auxins across the cells (Tanimoto, 2005; Taiz and Zeiger, 2010; Willige et al., 2011).

\section{PGPR: Microbial factories for auxin production}

Plant growth promoting rhizobacteria (PGPR) are the microbes present in close vicinity of the plants especially in the rhizosphere where these not only act as economical source of nutrition for the plants but improve soil fertility as well (Esitken et al., 2010; Malusa et al., 2012). Efficient bacterial colonization of the plant roots depends on the rhizospheric conditions conferred by the plant root exudates to the rhizosphere, microbial activities in response to root exudates and rhizospheric environment and mutual interactions of both rhizospheric bacterial communities and plant roots (Hartmann et al., 2009; Esitken et al., 2010; Lim 
et al., 2010). Root exudates secreted by the plant roots are microbe specific and are characterized by the ability to attract specific microbial species through chemotaxis thus restricting the colonization of the plant roots by specific bacterial communities out of the whole microbial population present in the rhizosphere. Root exudates can alter rhizospheric environment by altering $\mathrm{pH}$ and redox potential. Approximately $10-25 \%$ of the fixed carbon in majority of the plants are transported to their roots from where these are secreted in the form of root exudates containing organic and inorganic substances, sugars etc. (Glick et al., 1997). The availability and high concentrations of these nutrients in the root exudates is primarily responsible for the abundance of microorganisms in the rhizosphere as compared to the other soil areas away from plant roots. The majority of the plant growth promoting bacteria (PGPB) belong to the rhizospheric community rather than to the endophytic or phyllospheric bacterial population. Rhizospheric bacteria extract nutrients from the carbon compounds present in the root exudates, which act as energy source for them while the availability of nutrients to plants increases in response to the microbial activities through mobilization of insoluble minerals by processes as nitrogen fixation, siderophore formation, phosphate solubilization etc. In other words, substances secreted by plant roots act as energy source for the rhizospheric bacteria which help in facilitating mineral availability, water and nutrient uptake and secretion of plant growth promoting substances such as auxins or by acting as biocontrol agents thus preventing disease development and improving plant growth (Hartmann et al., 2009; Egorshina et al., 2012).

PGPR can be categorized into two classes which include those bacteria that beneficially affect plant through direct physiological or biochemical mechanisms and those PGPR which promote plant growth indirectly by reduction in the phytopathogenic strains and disease development thereby strengthening plant immune response i.e., PGPR acting as biocontrol agents (Glick, 1995; Glick et al., 1998; Rajendran et al., 2012). Different mechanisms such as water and nutrient uptake and changes in the root growth and development affected by rhizospheric bacteria are found to influence growth improvement of plants thus stimulation of multiple mechanisms by PGPB results in the overall promotion of plant growth (Lim et al., 2010; Meldau et al., 2012). It is speculated that rhizospheric bacteria stimulate stress-related protein synthesis thus helping the plants in tolerating various kinds of stresses (Lim et al., 2010). Presence of other microorganisms as well as different plant species affect the activity of PGPR so these may respond differently in different environments exhibiting variability in their affect on plant growth (Remans et al., 2008; Stajkovic et al., 2009). Evi- dence suggests that PGPR exhibiting beneficial impact on certain plants may not have any affect at all or may even inhibit growth in some other plant species (Khan and Doty, 2009).

Various mechanisms involved in growth improvement by PGPR include microbial phytohormone production (IAA, cytokinins, gibberellins), enhanced water and nutrient uptake, improved nitrogen availability in the soil, production of ACC deaminase for ethylene breakdown, phosphate solubilization, siderophore production etc. (Glick et al., 1998; Khan and Doty, 2009; Sgroy et al., 2009; Esitken et al., 2010; Mia et al., 2010; Kraiser et al., 2011; Rajendran et al., 2012). Biological nitrogen fixation and microbial production of phytohormones were observed to be the major factors responsible for plant growth improvement by PGPR, which help in the development of efficient root system for enhanced water and nutrient uptake (Mia et al., 2010; Meldau et al., 2012; Rajendran et al., 2012). Auxin production potential is not only limited to PGPRs but endophytic bacteria and plant growth promoting fungi are also known to produce auxins. The majority of the phytohormone producing bacteria have been found to produce IAA, although bacteria producing other phytohormones such as cytokinins and gibberellins have also been reported (Ortiz-Castro et al., 2008; Morrone et al., 2009). Since auxins act as master control affecting many plant processes, directly or indirectly, as well as interactively influence the synthesis and action of other phytohormones, therefore, the present review primarily focuses on auxin producing ability of rhizobacteria. Auxins secreted by bacteria have been found to act as signaling molecules for communication between bacteria to coordinate their activities (Ouzari et al., 2008). One of the most prominent features of plants inoculated with auxin-producing plant growth promoting bacteria is the modification in the root morphology and development. Thus, plant growth-promoting bacteria promote root growth by increasing root surface area which in turn promotes nutrient uptake thereby indirectly stimulating plant growth positively (Egorshina et al., 2012). There is increasing evidence that auxins are involved in regulating the expression of certain genes thus indirectly controlling many plant processes (Mia and Shamsuddin, 2010; Niklas and Kutschera, 2012).

Auxins secreted by PGPR activate biosynthetic signaling pathways (Roy et al., 2010). Since the level of auxins present in the plant environment critically affects plant development so it is speculated that microbial auxin production by PGPR may alter the level of auxins and affects all the physiological processes regulated by auxins thus promoting plant height, biomass and grain yield etc. (Molina-Favero et al., 2008; Khan and Doty, 2009). Most of the rhizospheric bacteria are able to produce IAA. These include pathogenic bacteria such 
as Erwinia herbicola, Agrobacterium tumefaciens, Agrobacterium rhizogenes and Pseudomonas syringae and plant growth promotiong bacteria such as Azotobacter sp., Pseudomonas sp., Azospirillum sp., Rhizobium sp., Bacillus sp. and Enterobacter sp. (Ouzari et al., 2008; Khan and Doty, 2009; Abd El-Hadi Nadia et al., 2009; Merzaeva and Shirokikh, 2010; Roy et al., 2010; Celloto et al., 2012). Plant pathogens may cause abnormal hormonal signaling leading to disease development in plants (Grant and Jones, 2009). IAA biosynthesis is not vital for bacterial growth as IAA deficient mutants were found to grow normally (Zakharova et al., 1999). The growth phase of auxin producing bacteria also plays critical role in determining the amount of IAA produced by the bacterial strains. Higher amounts of IAA are generally produced during stationary growth phase of bacteria (Ahmed and Hasnain, 2010). Azospirillum sp. are the nitrogen fixing bacteria that have the ability to produce IAA (Mia et al., 2010). IAA production by Azospirillum sp. is generally considered major factor for improving plant growth (Mehry et al., 2008; Kraiser et al., 2011). The effects observed by inoculation with Azospirillum brasilense include suppression of disease development, improved plant growth due to improved water and mineral absorption causing an increase in fresh and dry mass thereby increasing the plant yield (Mehry et al., 2008; Molina-Favero et al., 2008). Martinez-Morales et al. (2003) reported that A. brasilense produces IBA, a substance associated with auxin activity that stimulates plant growth. It has been observed that lateral and adventitious root formation is stimulated by inoculation with $A$. brasilense which also promotes the development of root hair thereby improving root system. Arora et al. (2001) reported that $96 \%$ of the rhizobial isolates produce IAA. Rhizobium sp. promotes plant growth even in dry conditions (Mia and Shamsuddin, 2010). Increased nodulation, better root development, greater yields, enhanced mineral uptake are the effects observed as a result of rhizobium inoculation (Patten and Glick, 2002; Mia and Shamsuddin, 2010; Gao et al., 2012). IAA deficient mutants of P. syringae were reduced in their ability to colonize roots (Arora et al., 2001).

\section{Mechanisms of microbial auxin production}

Bacteria synthesize IAA through several pathways, three of which are commonly observed i.e., IAM (indole-3-acetamide), IAN (indole-3-acetonitrile) and IPyA (indole-3-pyruvic acid) pathways (Fu and Wang, 2011; Niklas and Kutschera, 2012). Phytopathogenic bacteria synthesize IAA mainly by indoleacetamide pathway (Ouzari et al., 2008; Won et al., 2011). IAA synthesized by beneficial bacteria that promotes plant growth involves the ipdC gene which encodes indolepyruvate decarboxylase, an enzyme that catalyzes the conversion of IPyA to indole-3-acetaldehyde (Sgroy et al., 2009). In indoleacetamide pathway, which is the principle route of IAA production in phytopathogenic strains, the synthesis of indole-3-acetamide from tryptophan is catalyzed by tryptophan-2-monooxygenase. Similarly indoleacetamide hydrolase is the enzyme that catalyzes the conversion of indoleacetamide to IAA (Won et al., 2011) whereas in plant growth stimulating bacteria, synthesis of IAA proceeds through the intermediate indolepyruvic acid. In this pathway, tryptophan is converted to indole-3-pyruvic acid which is decarboxylated to indole-3-acetaldehyde by indole-3-pyruvate decarboxylase followed by IAA formation (Patten and Glick, 2002; Fu and Wang, 2011). Tryptophan (Trp), present in the root exudates, acts as a precursor of IAA and affects IAA synthesis in the majority of auxin producing PGPR (Merzaeva and Shirokikh, 2010). Almost 90\% reduction in IAA synthesis was observed in A. brasilense ipdC knock out mutants demonstrating the role of ipdC in Trp-dependent as well as Trp-independent IAA biosynthesis. It has also been reported that $90 \%$ of the IAA production by Azospirillum sp. follows Trp-independent pathway. However, increase in IAA production was recorded with elevated tryptophan levels (Brandl and Lindow, 1998; Molina-Favero et al., 2008). In P. syringae, IAA biosynthesis follow indoleacetamide pathway, however, in Pseudomonas fluorescens, Trp is converted directly to indole-3-acetaldehyde followed by the production of IAA. Auxin synthesis by PGPR does not always proceeds through tryptophan. Some PGPRs also synthesize IAA through mechanisms independent of tryptophan (Merzaeva and Shirokikh, 2010; Kiyohara et al., 2011). Studies revealed that bacterial IAA production is a pathway for the detoxification of tryptophan and auxins are secreted as secondary metabolites by the bacterial strains (Ouzari et al., 2008; Patil, 2011).

\section{Conclusion}

Plant growth promoting rhizobacteria (PGPR) are the plant associated bacteria frequently found in the rhizosphere of plants. Among the various mechanisms involved in plant growth enhancement by PGPR is microbial auxin production which has been reported to play major role in growth improvement of plants by PGPRs. Auxins produced by these bacteria trigger various metabolic processes thereby controlling various aspects of plant growth and development directly or indirectly. These activities finally lead to plant growth improvement. These auxin producing bacteria, with immense plant growth promotion potential owing to 
their ability to produce auxin, can therefore be efficiently utilized for plant growth improvement as an alternate to the chemical fertilizers which are imposing hazardous effects on human health.

\section{Literature}

Ahmed A. and S. Hasnain. 2010. Auxin producing Bacillus sp.: Auxin quantification and effect on the growth Solanum tuberosum. Pure Appl Chem. 82 (1): 313-319.

Abd El-Hadi Nadia I.M., H.K. Abo El-Ala and W.M. Abd ElAzim. 2009. Response of some Mentha species to plant growth promoting bacteria isolated from soil rhizosphere. Aust. J. Basic. Appl. Sci. 3(4): 4437-4448.

Acosta I.F. and E.E. Farmer E.E. 2010. Jasmonates. In: The Arabidopsis Book .10.1199/tab.0129: e0129.

Arora N.K., S.C. Kang and D.K. Maheshwari. 2001. Isolation of siderophore-producing strains of Rhizobium meliloti and their biocontrol potential against Macrophomina phaseolina that causes charcoal rot of groundnut. Curr. Sci. 81(6): 673-677.

Avanci N.C., D.D. Luche, G.H. Goldman and M.H.S. Goldman. 2010. Jasmonates are phytohormones with multiple functions, including plant defense and reproduction. Genet. Mol. Res. 9(1): 484-505.

Blomster T., J. Salojarvi, N. Sipari, M. Brosche, R. Ahlfors, M. Keinanen, K. Overmyer and J. Kangasjarvi. 2011. Apoplastic reactive oxygen species transiently decrease auxin signaling and cause stress-induced morphogenic response in Arabidopsis. Plant Physiol. 157: 1866-1883.

Brandl M.T. and S.E. Lindow. 1998. Contribution of Indole-3-acetic acid production to the epiphytic fitness of Erwinia herbicola. Appl. Environ. Microbiol. 64(9): 3256-3263.

Celloto V.R., A.J.B. Oliveira, J.E. Gonçalves, C.S.F. Watanabe, G. Matioli and R.A.C. Gonçalves. 2012. Biosynthesis of Indole-3-acetic acid by new Klebsiella oxytoca Free and immobilized cells on inorganic matrices. The Scientific World J. 2012 doi:10.1100/2012/495970

Chapman E.J., K. Greenham, C. Castillejo, R. Sartor, A. Bialy, T-p. Sun and M. Estelle. 2012. Hypocotyl transcriptome reveals auxin regulation of growth-promoting genes through GA-dependent and-independent pathways. PLoS ONE. 7(5): e36210. doi:10.1371/ journal.pone.0036210

Chiu R.S., H. Nahal, N.J. Provart and S. Gazzarrini. 2012. The role of the Arabidopsis FUSCA3 transcription factor during inhibition of seed germination at high temperature. BMC Plant Biol. 12(15). doi:10.1186/1471-2229-12-15.

Costigan S.E., S.N. Warnasooriya, B.A. Humphries and B.L. Montgomery. 2011. Root-localized phytochrome chromophore synthesis is required for photoregulation of root elongation and impacts root sensitivity to jasmonic acid in Arabidopsis. Plant Physiol. 157(3): 1138-1150.

Dempsey D.M.A., A.C. Vlot, M.C. Wildermuth and D.F. Klessig. 2011. Salicylic Acid biosynthesis and metabolism. In: The Arabidopsis Book. e0156. doi: 10.1199/tab.0156

Donner T.J., I. Sherr and E. Scarpella. 2010. Auxin signal transduction in Arabidopsis vein formation. Plant Signal. Behav. 5(1): 70-72. Egorshina A.A., R.M. Khairullin, A.R. Sakhabutdinova and M.A. Luk'yantsev. 2012. Involvement of phytohormones in the development of interaction between wheat seedlings and endophytic Bacillus subtilis strain 11BM. Russ. J. Plant Physl. 59(1): 134-140.

Esitken A., H.E. Yildiz, S. Ercisli, M.F. Donmez, M. Turan and A. Gunes. 2010. Effects of plant growth promoting bacteria (PGPB) on yield, growth and nutrient contents of organically grown Strawberry. Sci. Hortic. 124: 62-66.

Facella P., L. Daddiego, G. Giuliano and G. Perrotta. 2012. Gibberellin and Auxin influence the diurnal transcription pattern of photoreceptor genes via CRY1a in tomato. PLoS ONE. 7(1): e30121. doi:10.1371/journal.pone.0030121

Fu J. and S. Wang. 2011. Insights into auxin signaling in plantpathogen interactions. Front Plant Sci. 2: 1-7. doi: 10.3389/ fpls.2011.00074

Gao X., X. Lu, M. Wu, H. Zhang, R. Pan, J. Tian, S. Li and H. Liao. 2012. Co-inoculation with rhizobia and AMF inhibited soybean red crown rot: from field study to plant defense-related gene expression analysis. PloS ONE. 7(3): e33977. doi:10.1371/journal.pone.0033977 Giehl R.F.H., J.E. Limaand N. von Wiren. 2012. Localized iron supply triggers lateral root elongation in Arabidopsis by altering the aux1-mediated auxin distribution. Plant Cell. 24(1): 33-49.

Glick B.R. 1995. The enhancement of plant growth by free living bacteria. Can. J. Microbiology 41: 109-117.

Glick B.R., C. Liu, S. Ghosh and E.B. Dumbroff. 1997. Early development of canola seedlings in the presence of the plant growthpromoting rhizobacterium Pseudomonas putida GR12-2. Soil Biol. Biochem. 29(8): 1233-1239.

Glick B.R., D.M. Penrose and J. Li. 1998. A model for the lowering of plant ethylene concentrations by plant growth-promoting bacteria. J. theor. Biol. 190: 63-68.

Glick B.R. 2005. Modulation of plant ethylene levels by the bacterial enzyme ACC deaminase. FEMS Microbiology. 251: 1-7.

Grant M.R. and J.D.G. Jones. 2009. Hormone (Dis) harmony moulds plant health and disease. Science 324: 750

Hartmann A., M. Schimid, D.V. Tuinen, G. Berg. 2009. Plant driven selection of microbes. Plant Soil. 321: 235-257.

Hopkins W.G. and N.P.A. Huner. 2004. Introduction to Plant Physiology. $3^{\text {rd }}$ ed, John Wiley and Sons Inc., USA.

Jamil M., T. Charnikhova, B. Houshyania, A. Ast and H.J. Bouwmeester. 2012. Genetic variation in strigolactone production and tillering in rice and its effect on Striga hermonthica infection. Planta. 235(3): 473-484.

Kami C., M. Hersch, M. Trevisan, T. Genoud, A. Hiltbrunner, S. Bergmann and C. Fankhauser. 2012. Nuclear phytochrome a signaling promotes phototropism in Arabidopsis. Plant Cell. 24(2): 566-576.

Khan Z. and S.L. Doty. 2009. Characterization of bacterial endophytes of sweet potato plants. Plant Soil. 1-7. doi: 10.1007/ s11104-009-9908-1

Kiyohara S., H. Honda, N. Shimizu, C. Ejima, R. Hamasaki and S. Sawa. 2011. Tryptophan auxotroph mutants suppress the superroot 2 phenotypes, modulating IAA biosynthesis in Arabidopsis. Plant Signal. Behav. 6(9):1351-1355.

Koltai H. 2011. Strigolactones' ability to regulate root development may be executed by induction of the ethylene pathway. Plant Signal. Behav. 6(7): 1004-1005.

Kraiser T., D.E. Gras, A.G. Gutierrez, B. Gonzalez, and R.A. Gutierrez. 2011. A holistic view of nitrogen acquisition in plants. J. Exp. Bot. 62(4): 1455-1466.

Lim K-A., Z.H. Shamsuddin and C-L. Ho. 2010. Transcriptonic changes in the root of oil palm (Elaecis guineensis Jacq.) upon inoculation with Bacillus sphaericus UPMB10. Tree Genet. Genomes 6 : 793-800.

Liu H., X. Li, J. Xiao and S. Wang. 2012. A convenient method for simultaneous quantification of multiple phytohormones and metabolites: application in study of rice-bacterium interaction. Plant Methods. 8(2): doi: 10.1186/1746-4811-8-2

Malusa E., L. Sas-Paszt and J. Ciesielska. 2012. Technologies for beneficial microorganisms inocula used as biofertilizers. The Scientific World J. 2012. doi:10.1100/2012/491206 
Martinez-Morales L.J., L. Soto-Urzua, B.E. Baca, J.A. SanchezAhedo. 2003. Indole-3-butyric acid (IBA) production in culture medium by wild strain Azospirillum brasilense. FEMS Microbiol. Lett. 228(2): 167-173.

Mehry A., M. Akbar and E. Giti. 2008. Colonization and nitrogenase activity of Triticum aestivum (cv. Baccross and Mahdavi) to the dual inoculation with Azospirillum brasilense and Rhizobium meliloti plus 2,4-D. Pak. J. Biol. Sci. 11(12): 1541-1550

Meldau D.G., H.H. Long and I.T. Baldwin. 2012. A native plant growth promoting bacterium, Bacillus sp. B55, rescues growth performance of an ethylene-insensitive plant genotype in nature. Front Plant Sci. 3 doi: 10.3389/fpls.2012.00112

Merzaeva D.V. and I.G. Shirokikh. 2010. The production of auxins by the endophytic bacteria of winter rye. Appl. Biochem. Microbiol. 46(1): 44-50.

Mia M.A.B. and Z.H. Shamsuddin. 2010. Rhizobium as a crop enhancer and biofertilizer for increased cereal production. Afr. J. Biotechnol. 9(37): 6001-6009.

Mia M.A.B., Z.H. Shamsuddin and M. Mahmood. 2010. Use of plant growth promoting bacteria in banana production: A new insight for sustainable banana production. Int. J. Agr. Biol. 12(3): 459-467.

Molina-Favero C., C.M. Creus, M. Simontachi, S. Puntarulo and L. Lamattina. 2008. Aerobic nitric oxide production by Azospirillum brasilense Sp245 and its influence on root architecture in tomato. Mol Plant-Microbe Interact. 21(7): 1001-1009.

Morrone D., J. Chambers, L. Lowry, G. Kim, A. Anterola, K. Bender and R.J. Peters. 2009. Gibberellin biosynthesis in bacteria: Separate ent-copalyl diphosphate and ent-kaurene synthases in Bradyrhizobium japonicum. FEBS Letters. 583: 475-480

Muday G.K., A. Rahman and B.M. Binder. 2012. Auxin and ethylene: collaborators or competitors? Trends Plant Sci. 17(4): 181-195. Müller M. and S. Munné-Bosch. 2011. Rapid and sensitive hormonal profiling of complex plant samples by liquid chromatography coupled to electrospray ionization tandem mass spectrometry. Plant Methods. 7(37) doi:10.1186/1746-4811-7-37.

Niklas K.J. and U. Kutschera. 2012. Plant Development, auxin, and the subsystem incompleteness theorem. Front Plant Sci. 3. doi: 10.3389/fpls.2012.00037

Ortiz-Castro R., Valencia-Cantero E. and J. Lopez-Bucio. 2008. Plant growth promotion by Bacillus megaterium involves cytokinin signalling. Plant Signal. Behav. 3:4: 263-265.

Ouzari H., A. Khsairi, N. Raddadi, L. Jaoua, A. Hassen, M. Zarrouk, D. Daffonchio and A. Boudabous. 2008. Diversity of auxinproducing bacteria associated to Pseudomonas savastanoi-induced olive knots. J. Basic Microbiol. 48: 370-377.

Patil V. 2011. Production of indole aetic acid by Azotobacter sp. Recent Research Sci. Technol. 3(12): 14-16.

Patten C.L. and B.R. Glick. 2002. Role of Pseudomonas putida Indoleacetic acid in development of the host plant root system. Appl. Environ. Microbiol. 68(8): 3795-3801.

Pierik R., T. Djakovic-Petrovic, D.H. Keuskamp, M. de Wit and A.C.J. Voesenek. 2009. Auxin and Ethylene regulate elongation responses to neighbor proximity signals independent of gibberellin and DELLA proteins in Arabidopsis. Plant Physiology. 149: 1701-1712.
Rajendran G., M.H. Patel and S.J. Joshi. 2012. Isolation and characterization of nodule-associated Exiguobacterium sp. from the root nodules of fenugreek (Trigonella foenum-graecum) and their possible role in plant growth promotion. Int. J. Microbiol. doi:10.1155/2012/693982

Remans R., S. Beebe, M. Blair, G. Manrique, E. Tovar, I. Rao, A. Croonenborghs, R. Torres-Gutierrez, M. El-Howeity, J. Michiels and J. Vanderleyden. 2008. Physiological and genetic analysis of root responsiveness to auxin-producing plant growth-promoting bacteria in common bean (Phaseolus vulgaris L.) Plant Soil. 302: 149-161

Robles L.M., S.D. Deslauriers, A.A. Alvarez and P.B. Larsen. 2012. A loss-of-function mutation in the nucleoporin AtNUP160 indicates that normal auxin signalling is required for a proper_ethylene response in Arabidopsis. J. Exp. Bot. 63(5): 2231-2241.

Roy B.D., B. Deb and G.D. Sharma. 2010. Role of acetic acid bacteria in biological nitrogen fixation. Biofrontiers. 1(2): 83-98.

Ruzicka K., K. Ljung, S. Vanneste, R. Podhorska, T. Beeckman, J. Frimi and E. Benkova. 2007. Ethylene regulates root growth through effects on auxin distribution. Plant Cell. 19:2197-2212.

Sgroy V., F. Cassan, O. Masciarelli, M.F.D. Papa, A. Largares and V. Luna. 2009. Isolation and characterization of endophytic plant growth promoting (PGPB) or stress homoeostasis-regulating (PSHB) bacteria associated to the halophytes Prosopis strombulifera. Appl. Microbiol. Biotechnol. doi 10.1007/s00253-009-2116-3

Smith R.S. 2008. The role of auxin transport in plant patterning mechanism. PloS Biol. 6(12):2631-2633.

Stajkovic O., S.D. Meyer, B. Milicic, A. Willems and D. Delic. 2009. Isolation and characterization of endophytic non-rhizobial bacteria from root nodules of alfalfa (Medicago sativa L.). Bot. Serb. 33(1): 107-114.

Stewart J.L. and J.L. Nemhauser. 2010. Do trees grow on money? Auxin as the currency of the cellular economy. Cold Spring Harb Perspect Biol. 2. a001420. doi: 10.1101/cshperspect.a001420

Taiz L. and E. Zeiger. 2010. Plant Physiology fifth Edition. Sinauer Associates., Inc. 23 Plum tree Road, Sunderland, MA 01375, USA. Tanimoto E. 2005. Regulation and root growth by plant hormonesroles for auxins and gibberellins. Critical Rev. Plant. Sci. 24: 249-265. Teale W.D., F.A. Ditengou, A.D. Dovzhenko, X. Li, A.M. Molendijk, B. Ruperti, I. Paponov and Palme K. 2008. Auxin as a Model for the integration of hormonal signal processing and transduction. Molecular Plant. 1(2): 229-237.

Willige B.C., E. Isono, R. Richter, M. Zourelidou and C. Schwechheimer. 2011. Gibberellin regulates PIN-FORMED abundance and is required for auxin transport-dependent growth and development in Arabidopsis thaliana. Plant Cell. 23: 2184-2195.

Won C., X. Shen, K. Mashiguchi, Z. Zheng, X. Dai, Y. Cheng, H. Kasahara, Y. Kamiya, J. Chory, Y. Zhao Y. 2011. Conversion of tryptophan to indole-3-acetic acid by tryptophan aminotransferases of Arabidopsis and YUCCAS in Arabidopsis. PNAS 108(45): 18518-18523.

Wu H-m., O. Hazak, A.Y. Cheung and S. Yalovsky. 2011. RAC/ ROP GTPases and auxin signaling. Plant Cell. 23: 1208-1218.

Zakharova E.A., Shcherbakov A.A., Brudnik V.V., Skripko N.G., Bulkhin N.S. and V.V. Ignatov. 1999. Biosynthesis of Indole-3-acetic acid in Azospirillum brasilense. Eur. J. Biochem. 259(3): 572-576. 